\title{
Algo de historia en los 65 años de fundación de la Facultad de Odontología de la Pontificia Universidad Javeriana: El "Nuevo Currículo" (1985-2005) y la Unidad de Investigación y Tecnología Educativa
}

\author{
A Piece of History in the 65 th Anniversary of Foundation of the \\ Javeriana University Dental School: The "New Curriculum" \\ (1985-2005) and the Department of Dental Research and Education
}

\begin{abstract}
Jorge Enrique Delgado Troncoso
Odontólogo (promoción junio de 1989), magíster en Educación, docente (enero 1990-enero 2005), Pontificia Universidad Javeriana, Bogotá, Colombia. PhD en Análisis Social y Comparado en Educación, docente de educación y temas latinoamericanos, Universidad de Pittsburgh, Estados Unidos. Editor general de Universitas Odontologica (2003-2005, enero del 2009 al presente).
\end{abstract}

CÓMO CITAR ESTE ARTíCULO Delgado JE. Algo de historia en los 65 años de fundación de la Facultad de Odontología

de la Pontificia Universidad Javeriana: el "Nuevo Currículo" (1985-2005) y la Unidad de Investigación y Tecnología Educativa. Univ Odontol. 2015 Jul-Dic; 34(73): 15-19. http:// dx.doi.org/10.11144/Javeriana.uo34-73.haff

doi: 10.11144/Javeriana.uo34-73.haff
El presente artículo se escribió como un aporte al libro que se publicó para celebrar los 65 años de fundación de la Facultad de Odontología de la Pontificia Universidad Javeriana (1). El libro incluye algunos apartes del manuscrito y aquí se publica en su totalidad con algunos ajustes. Representa la experiencia del autor como miembro de la otrora Unidad de Investigación y Tecnología Educativa, unidad académica que sirvió de apoyo y lideró el desarrollo del proyecto curricular del programa de pregrado que se implementó entre 1985 y 2005.

El currículo del pregrado en Odontología de la Pontificia Universidad Javeriana se ha transformado dos o tres veces durante la historia de la Facultad de Odontología. Una de las reformas curriculares más importantes tuvo lugar a mediados de los años ochenta. En ese tiempo comenzó un proceso de cambio que incluyó una reconceptualización de la odontología y que concluyó no solo con un cambio curricular, sino también con una reestructuración administrativa de toda la Facultad. Entre los impulsores de este proceso estuvieron profesores como Nelson Contreras Caballero y Enrique Echeverri Guzmán.

La reforma de la década de los ochenta se basó en las siguientes perspectivas conceptuales que se denominaron enfoques: enfoque por problemas odontológicos, enfoque por sistemas, enfoque epidemiológico, enfoque por dimensiones y enfoque por objetivos de aprendizaje. De acuerdo con el enfoque por problemas odontológicos, se identificaron los cuatro principales grupos de enfermedades bucales que afectan a la población: la caries dental, las enfermedades periodontales, las anomalías dentomaxilofaciales y las patologías de tejidos blandos y duros, junto con aquellas que generan o provienen de alguna afectación de tipo sistémico. El enfoque por sistemas tomó la teoría de sistemas para identificar las estructuras bucales involucradas en cada problema odontológico, las dinámicas de los procesos de salud-enfermedad y los factores asociados a ellos. Así, se establecieron cuatro sistemas: Dentario, Periodontal, Craneofacial y Bucal. Ellos originaron los departamentos de los sistemas que existen en la actualidad y que remplazaron los tradicionales departamentos por especialidades odontológicas. Las especialidades fueron absorbidas por los departamentos: Endodoncia, 
Prostodoncia y Operatoria se incorporaron a Dentario; Periodoncia, Preventiva y Social se unieron a Periodontal; Odontopediatría, Ortodoncia y Articulación Temporomandibular entraron a formar parte de Craneofacial; Imagenología, Farmacología y Terapéutica, Patología, Semiología y Cirugía se agruparon en Bucal. Esta redefinición y restructuración no dejó de atraer críticos, pero se impuso por su novedad. Esa estructura administrativa se ha mantenido con algunas reformas menores en el interior de los departamentos en años más recientes. Los departamentos también coordinan académicamente los posgrados, según la afinidad temática.

Ahora bien, para el enfoque epidemiológico se tomaron como modelos la Historia Natural de la Enfermedad (HNE) y los Niveles de Prevención, de Leavell y Clark de 1958. La HNE identifica tres grandes periodos del proceso patogénico e indica cómo se van interrelacionando los componentes etiológicos de la tríada epidemiológica: el huésped, el agente y el ambiente. En el periodo prepatogénico existen las condiciones generales (sociales o ambiente propicio general) y específicas (factores de riesgo o ambiente propicio específico) para que se produzca una enfermedad. Durante el periodo patogénico ya se ha establecido la enfermedad y puede ser imperceptible en el examen clínico (etapa subclínica, identificable a través de medios de diagnóstico) o ya haberse manifestado (etapa clínica, donde ya existen signos y síntomas). El periodo pospatogénico (etapa de secuelas) tiene lugar cuando la enfermedad ha llegado a sus últimas consecuencias y ha producido secuelas: la pérdida de un órgano o incluso la muerte. Los niveles de prevención primaria, secundaria y terciaria son las intervenciones que se efectúan para contrarrestar los periodos de la enfermedad y sus etapas mediante estrategias y actividades de promoción de la salud, prevención específica, diagnóstico precoz y tratamiento oportuno, limitación del daño y rehabilitación.

El enfoque epidemiológico es muy importante, porque el plan de estudios del pregrado de Odontología fue estructurado utilizando la HNE y los niveles de prevención. Así, los estudiantes aprendían en los primeros semestres sobre el periodo prepatogénico y la prevención primaria en cada uno de los sistemas e iban aumentando en complejidad a medida que avanzaban en la carrera. Un aspecto muy innovador ligado a este enfoque, emulado por otras facultades de odontología, fue la creación de prácticas clínicas desde el primer semestre. Los estudiantes en los semestres iniciales acudían a las Clínicas del Sano, diseñadas con base en el modelo que creó y divulgó el doctor Benjamín Herazo Acuña hacia 1984 y que habían mostrado ser efectivas para prevenir la aparición de enfermedades bucales por medio de acciones de prevención primaria.

El enfoque por dimensiones miraba al ser humano integralmente y expresado en cuatro áreas o dimensiones. Así, el currículo concebía al hombre como ser biogenético, histórico-social, psicoafectivo y trascendente. Dichas dimensiones permitían la colaboración de Odontología con otras facultades y departamentos de la universidad.

De la mano del nuevo modelo curricular, que implicaba un cambio radical en la forma como se definía y enseñaba la odontología, se desarrolló también un modelo de enseñanza-aprendizaje a partir de los principios de la tecnología educativa y los modelos pedagógicos imperantes de esa época: el enfoque por objetivos. Para ello, se identificaron las grandes metas de aprendizaje en la formación del odontólogo, algo parecido a las competencias que se vienen empleando desde comienzos del siglo XXI, y se crearon estructuras de aprendizaje que incluían objetivos específicos de aprendizaje para cada asignatura en cada semestre. Los objetivos en dichas estructuras estaban organizados por conductas y niveles cognoscitivos de aprendizaje en orden ascendente de complejidad. Se utilizaron 
para ello las taxonomías de Bloom y Gagné de los años setenta que incluían memoria, comprensión, aplicación, análisis, síntesis y evaluación. Como se puede inferir, fue necesario crear la infraestructura y conformar un equipo humano que se encargara del desarrollo, de la implementación y de la evaluación del nuevo modelo curricular. Surgió así la Unidad de Investigación y Tecnología Educativa.

Entre los primeros miembros de la "Unidad" estuvieron el doctor Nelson Contreras, quien lideró la conceptualización, la implementación y la coordinación del proyecto; la tecnóloga educativa Lina Claricia Corredor, quien se encargó de crear el modelo pedagógico, y el ingeniero Luis Alberto Martínez, quien era el responsable del sistema de información y la red. Con el tiempo, se vincularon a la Unidad la enfermera Aurora Montoya y el odontólogo John Harold Estrada, quienes estudiaron maestrías en Educación Comunitaria, para continuar con el desarrollo del currículo y coordinar los talleres de capacitación a los profesores y la escritura de las estructuras y guías de aprendizaje. Estas últimas eran otro componente de la estrategia de enseñanza-aprendizaje y se elaboraban siguiendo el Modelo Didáctico Operativo, creado por Félix Bustos en los años ochenta. Las guías tomaban elementos de diferentes perspectivas pedagógicas, como la pedagogía activa, e incluían momentos o etapas de vivencia (experimentación), conceptualización, teorización, ampliación, aplicación y valoración. Se buscaba con ello pasar de la didáctica basada en la transmisión y la memorización de contenidos a una enseñanza en la cual el estudiante construye su propio conocimiento con la orientación del docente. Podían existir una o más guías de aprendizaje en cada materia. El "nuevo currículo" comenzó a implementarse de modo gradual con la cohorte de estudiantes que inició en enero de 1985. Las estructuras de aprendizaje, las guías de aprendizaje y la capacitación de los docentes y administrativos se fueron desarrollando de manera escalonada con el apoyo de la Unidad de Investigación.

Otra de las innovaciones del nuevo currículo fue el énfasis en investigación. Se estableció que los estudiantes de tercer semestre que tenían práctica en comunidades de niveles socioeconómicos bajos debían desarrollar proyectos grupales de investigación descriptiva a partir de su experiencia en salud pública y odontología preventiva con esas comunidades. La enseñanza de la investigación era también responsabilidad de la Unidad de Investigación. La profesora de la clase de metodología entre 1988 y el 2000 fue la socióloga magistra en Educación Alegría Rincón de Galvis. Poco a poco, Alegría se convirtió en una de las personas más influyentes que hayan pasado por la Facultad de Odontología por su visión, liderazgo, inteligencia, elocuencia y buenas relaciones humanas. Ella sucedió al doctor Néstor Contreras en la dirección de la Unidad de Investigación, cuando él fue nombrado Decano Académico de la Facultad, en 1994.

Otra de las estrategias para desarrollar la investigación consistió en requerir que todos los estudiantes condujeran algún tipo de estudio como trabajo de grado. Hasta ese momento, la mayor parte de las "tesis" de los estudiantes de pregrado habían sido monografías. El nuevo proceso de investigación ponía el relieve en la escritura y aprobación de cuatro documentos por parte de la Unidad y los departamentos: anteproyecto, protocolo, informe de avance y tesis. En sexto semestre, los estudiantes comenzaban con el anteproyecto, que incluía el planteamiento del problema y la formulación de las preguntas de investigación, así como con el propósito, los objetivos y la justificación del estudio. El protocolo se presentaba en séptimo semestre y adicionaba a los elementos del anteproyecto el marco de referencia y el diseño de la investigación. Los semestres octavo y noveno se destinaban a la recolección y al análisis de la información. El documento de esta fase era el informe de avance. Por último, la tesis o documento final agregaba los resultados, la discusión, 
las conclusiones y las recomendaciones. Además de la aprobación del documento, los protocolos y tesis eran defendidos durante la semana de sustentaciones que tenía lugar cada semestre. Una vez un protocolo o una tesis eran aprobados, los estudiantes debían escribir un artículo científico que, en la mayoría de las ocasiones, era sometido a evaluación para publicación en la revista científica de la facultad: Universitas Odontologica. Cada año, la facultad seleccionaba los mejores trabajos para presentarlos en el Encuentro de Investigación de la Asociación Colombiana de Facultades de Odontología (ACFO).

La investigación del pregrado cobró tal importancia y prestigio que llevó a una época dorada de la investigación en la Facultad. Por medio de la dirección de tesis, muchos docentes vieron la oportunidad de aprender e involucrarse en proyectos de investigación y, en algunos casos, crearon líneas de investigación. Los miembros de la Unidad de Investigación tenían como responsabilidad evaluar metodológicamente los documentos, acompañar a los estudiantes en el proceso y realizar los trámites en los respectivos departamentos. Con el tiempo, la Unidad comenzó también a hacerse cargo del proceso metodológico de los trabajos de las especialidades y a colaborar de esta manera con la Unidad de Posgrados, Educación Continuada y Programas Especiales.

Además del desarrollo curricular y la administración de la investigación, la Unidad de Investigación asesoraba la elaboración de los exámenes, lideraba los procesos de autoevaluación para la acreditación universitaria, coordinaba la elaboración de las preguntas por parte de la Javeriana para los Exámenes de la Calidad de la Educación Superior (ECAES) y llevaba a cabo los talleres de capacitación docente en educación e investigación. Algunos títulos de estos talleres fueron Pedagogía Activa, Investigación como Eje del Currículo en Odontología, Razón de Ser del Maestro, Currículo Académico, Dirección de Proyectos de Investigación, Métodos y Diseño de Investigaciones, Estrategias Pedagógicas y Evaluación Educativa. Los miembros de la Unidad también representaban a la Facultad en las Divisiones de Investigación y Educación de ACFO.

Varios docentes estuvieron vinculados a la Unidad de Investigación y Tecnología Educativa durante el tiempo que existió. Después de la partida en diferentes momentos de Lina Claricia Corredor, Aurora Montoya y John Harold Estrada, por allí pasaron el profesor de estadística Adolfo Rico Angarita y los odontólogos Claudia Patricia Rivero, Gloria Cristina Moreno, Juan Fernando Yepes, Jorge Arturo Rey, Jorge Enrique Delgado, entre otros. Después del fallecimiento de la doctora Alegría Rincón, en el 2001, asumió como directora de la Unidad la doctora María Beatriz Ferro. De la mano de la doctora Ferro, la Unidad lideró desde comienzos del siglo XXI un nuevo proceso de reforma curricular. Como la Unidad de Investigación y Tecnología Educativa era un ente atípico dentro de la estructura organizacional de la Pontificia Universidad Javeriana, a comienzos de la primera década del siglo XXI se convirtió en el Comité de Carrera y sus responsabilidades se redefinieron y reasignaron.

La Unidad de Investigación y Tecnología Educativa quedó atrás, pero es claro que durante más de quince años su existencia marcó un hito en la historia de la Facultad de Odontología. Básicamente, la Unidad se ocupó por años de muchas de las áreas para los cuales los odontólogos clínicos y los departamentos no estaban preparados. Apoyó el diseño, la implementación y la evaluación del currículo, coordinó metodológicamente la evaluación, desarrolló la primera fase de la investigación de la Facultad, capacitó a los profesores en investigación y docencia, llevó a cabo los primeros procesos de autoevaluación para acreditación, entre 
otras actividades. Para muchos de los que tuvimos la suerte de trabajar en la Unidad, esta fue una experiencia interdisciplinaria única, enriquecedora y transformadora a tal punto que varios de nosotros dedicamos nuestras carreras profesionales al trabajo en educación en los ámbitos universitario y comunitario.

\section{REFERENCIA}

Villegas Solórzano V. (editora). 65 años Facultad de Odontología. Bogotá: Pontificia Universidad Javeriana, Facultad de Odontología; 2015.

\section{CORRESPONDENCIA}

Jorge Enrique Delgado Troncoso

jdelgado4501@yahoo.com.ar

universitas.odontologica@gmail.com 
